\title{
CONTEMPORARY FRACTION OF BIS(2-ETHYLHEXYL) PHTHALATE IN STILTON CHEESE BY ACCELERATOR MASS SPECTROMETRY
}

\author{
Michael A Nelson ${ }^{1,2} \cdot$ John M Ondov $^{1} \bullet$ Michael C VanDerveer $^{3} \bullet$ Bruce A Buchholz $^{4}$ \\ ABSTRACT. Measurements of the radiocarbon abundance in 5 samples of bis(2-ethylhexyl) phthalate (DEHP) isolated \\ from Stilton cheese were made by accelerator mass spectrometry (AMS) to determine the fraction of carbon originating from \\ contemporary biogenic sources. DEHP is classified as a "priority hazardous substance" by the European Union, a probable \\ human carcinogen by the United States Environmental Protection Agency, and is suspected to be a human endocrine dis- \\ rupter. Measurement of its ${ }^{14} \mathrm{C}$ abundance in a specific food indicates whether its presence is due to contamination from indus- \\ trially synthesized DEHP or a naturally inherent component. A method was developed to determine the contemporary carbon \\ fraction of DEHP in a fatty food matrix at concentrations of $\approx 0.14 \mathrm{mg} / \mathrm{kg}$. Five $90-\mu \mathrm{g}$ quantities of DEHP were extracted from \\ $12 \mathrm{~kg}$ of Stilton cheese and isolated by silica gel, size exclusion, and high-performance liquid chromatography (HPLC). \\ Masses of samples were determined by gas chromatography mass spectrometry (GC-MS) analyses prior to combustion and \\ manometry afterwards. The purity of DEHP carbon mass in each isolate was determined by multivariate deconvolution of \\ GCMS fragmentation spectra obtained from measurements of standards and isolates, and ranged from $88.0 \pm 1.8 \%$ to $92.3 \pm$ \\ $1.1 \%(n=5,1 \sigma)$. Concurrently processed isolation method blanks contained from $0.15 \pm 0.04$ to $1.52 \pm 0.06 \mu \mathrm{g}(n=3,1 \sigma)$ \\ DEHP per sample and significant quantities of pre- and post-chromatographic extraneous carbon contamination. The mean \\ ${ }^{14} \mathrm{C}$-corrected contemporary carbon fraction of DEHP in the isolates was $0.235 \pm 0.073(1 \sigma$; and \pm 0.091 at the $95 \%$ confi- \\ dence level), revealing that the majority of DEHP in Stilton cheese results from anthropogenic sources, but with a significant \\ naturally occurring component.
}

\section{INTRODUCTION}

Phthalic acid esters (PAE, i.e. 1,2-benzenedicarboxylic acids), also known as phthalates, are used in the manufacturing of many consumer and industrial products including pharmaceuticals, plastic polymers, adhesives, inks, and cosmetics (Schettler 2006). Bis(2-ethylhexyl) phthalate (DEHP) comprises half of the estimated 8 million tonne annual industrial phthalate production(Wenzl 2009). Leaching of this compound from many plastic consumer products ultimately leads to its environmental ubiquity and exposure (Schettler 2006).

Despite its low acute toxicity, DEHP has been classified a probable human carcinogen (US EPA 1997) and is suspected to be a human endocrine disruptor that mimics estradiol, causing irregular development and feminization in young boys (Jarosova 2006) and possibly decreased fertility in men and in women of child-bearing age (Zhu et al. 2006; David 2000). Accordingly, some developed nations, including the US and those in the European Union, have phased out the use of phthalates in food-contact materials (Enneking 2006) due to their proclivity for leaching into fatty foods (Castle et al. 1990). Human dietary consumption has been identified as a significant source of DEHP (Wormuth et al. 2006) and possibly the single most likely route of exposure to the general populace (Fromme et al. 2004; Schettler 2006). Unlike many other phthalate-containing materials, such as cosmetics and certain plastic products, the consumption of food is a necessary and unavoidable route to human exposure.

A comprehensive European study of DEHP in food reports that concentrations in non-dairy beverages averaged $0.01-0.04 \mathrm{mg} / \mathrm{kg}$; non-fatty foods such as fruit, vegetables, and grain products, $0.01-$

\footnotetext{
${ }^{1}$ Department of Chemistry and Biochemistry, University of Maryland, College Park, Maryland 20742, USA.

${ }^{2}$ Analytical Chemistry Division, Material Measurement Laboratory, National Institute of Standards and Technology,

Gaithersburg, Maryland 20899, USA. Corresponding author. Email: manelson@umd.edu.

${ }^{3}$ US Food and Drug Administration, University Station, College Park, Maryland 20740 USA.

${ }^{4}$ Center for Accelerator Mass Spectrometry, Lawrence Livermore National Laboratory, Livermore, California 94551, USA.
}

(C) 2013 by the Arizona Board of Regents on behalf of the University of Arizona

Proceedings of the 21st International Radiocarbon Conference edited by A J T Jull \& C Hatté

RADIOCARBON, Vol 55, Nr 2-3, 2013, p 686-697 


\section{MA Nelson et al.}

$0.57 \mathrm{mg} / \mathrm{kg}$; and fatty foods such as oils, dairy, animal, and nut products, $0.22-1.45 \mathrm{mg} / \mathrm{kg}$ (Wormuth et al. 2006). These concentrations were consistently greater than those observed in 6 other phthalates in the same food matrices, which is not surprising considering the much larger industrial production of DEHP. These values support the contention that phthalates are typically more concentrated in foods with a high lipid component and that DEHP is the most abundant of these compounds in commonly consumed foods. Screening analyses performed in our laboratories prior to work described herein found DEHP to account for most of the PAE content in various cheeses at mass fractions of $0.20-0.41 \mathrm{mg} / \mathrm{kg}$ (M Nelson, unpublished data).

Although contamination by anthropogenic phthalates may continue to occur, evidence of naturally produced phthalates has been reported for several types of algae and fungus. Chen (2004) demonstrated the biosynthesis of DEHP by red algae (Bangia atropurpurea) grown in a culture medium containing ${ }^{14} \mathrm{C}$-labeled sodium bicarbonate. More recently, Namikoshi et al. (2006) determined the modern carbon content of DEHP isolated from 3 different algae ( 2 of which are edible) to range from $50 \%$ to $87 \%$. Previously, Amade et al. (1994) had determined that DEHP comprised $23 \%$ of the ethanol-extracted compounds from fungal culture broths of Penicillium olsonii, and suggested it to be a metabolite of the fungus. Many algae products are used as food additives, and penicillium is often used as a fermentation agent in foods such as blue cheese. That DEHP can be synthesized by certain types of algae and mold used in food suggests that they are potentially important additional sources of dietary DEHP exposure, especially in light of the reduction of DEHP in food-contact materials. Therefore, the United States Food and Drug Administration (US FDA) is interested in determining the origin of DEHP in food, particularly fatty foods.

Synthesis of DEHP by biological sources likely amalgamates atmospheric steady-state ${ }^{14} \mathrm{C}$ from the biological origins of the food matrix from which it is produced. Petroleum formed from organic matter that has been isolated from atmospheric ${ }^{14} \mathrm{CO}_{2}$ for millions of years has a ${ }^{14} \mathrm{C}:{ }^{12} \mathrm{C}$ ratio that has decayed to immeasurably low levels (Namikoshi et al. 2006). Synthetic, anthropogenic DEHP used in plastics is produced from a 2-step esterification of petrogenic phthalic anhydride with petrogenic ethylhexanol (ECPI 2012), and thus is also devoid of measurable ${ }^{14} \mathrm{C}$. Therefore, measurements of ${ }^{14} \mathrm{C}:{ }^{12} \mathrm{C}$ in DEHP content in food will reveal whether it is naturally produced by biosynthesis from contemporary carbon sources or if more can be done to reduce anthropogenic environmental and food-processing contamination.

Compound-specific radiocarbon analysis (CSRA) has been performed to determine the age and origin of $<100-\mu \mathrm{g}$ quantities of compounds similar to DEHP, including polycyclic aromatic hydrocarbons (PAH) ranging in concentration from 0.03 to $10 \mathrm{mg} / \mathrm{kg}$ in several environmental Standard Reference Materials (Reddy et al. 2002) and lipid biomarkers at $\geq 1 \mathrm{mg} / \mathrm{kg}$ concentrations (Pearson et al. 2001) in marine sediments. There have been no ${ }^{14} \mathrm{C}$ measurements of phthalates in processed food and, most notably, none in fatty foods. It was a goal of the US FDA to obtain ${ }^{14} \mathrm{C}$ data on food matrices. For this purpose, Stilton cheese was selected for purification and ${ }^{14} \mathrm{C}$ analyses. Stilton cheese was selected due to its high fat content (35\% mass fraction) and because Stilton cheese, like other blue Roquefort cheeses, is injected with cultures of Penicillium roqueforti, which might also be expected to synthesize DEHP from contemporary compounds in the cheese.

Although single compound analyses of as little as $10 \mu \mathrm{g}$ of carbon have been reported, and the Lawrence Livermore National Laboratory Center for Accelerator Mass Spectrometry (LLNL CAMS) routinely performs analyses on samples as small as $20 \mu \mathrm{g}$ carbon, $100-\mu \mathrm{g}$ masses are preferable when supply of the sample is not a limiting factor. Accordingly, we sought to develop a method to isolate 70-100 $\mu \mathrm{g}$ quantities of DEHP. As the Stilton cheese analyzed in our work con- 
tained as little as $0.12 \mathrm{mg} / \mathrm{kg}$ DEHP, a 7.5 -million-fold enrichment was required to obtain these quantities at $90 \%$ purity.

\section{METHODS}

\section{Samples and Extraction}

Two 7-kg wheels of Stilton cheese (Neal's Yard Borough Market brand, London, UK) were purchased from a Whole Foods Market (Gaithersburg, MD, USA) and received in the manufacturer's original paper packaging for use in this study. Twelve kilograms of cheese was extracted in seven $1.2-2.6 \mathrm{~kg}$ batches to isolate enough DEHP to provide five 70- to $100-\mu \mathrm{g}$ samples of carbon for analysis by accelerator mass spectrometry (AMS). In addition, 4 method blanks were prepared contemporaneously (see Table 1) using only the extractants, eluents, and diluents used in the sample extraction-purification procedure. A fully deuterated (d38) DEHP phthalate standard (98\% pure, Cambridge Isotope Laboratories, Andover, MA, USA) was used to spike the raw cheese matrix in order to determine method yield and act as a resolvable chromatographic reference for unlabeled DEHP during the purification steps. The 4 method blanks were likewise spiked. Cheese samples were cut vertically from the wheels to ensure uniformity of the sampling location, homogenized, and sequentially extracted 3 times by manually stirring with 3 different solvents at $40{ }^{\circ} \mathrm{C}(2400 \mathrm{~mL}$ hexanes; $1200 \mathrm{~mL}$ 5:1 volume fraction hexane:acetone; and $800 \mathrm{~mL}$ hexanes). The 3 extracts were combined, evaporated to $2 \mathrm{~L}$, and centrifuged at $66,850 \mathrm{rad} / \mathrm{s}$ for $10 \mathrm{~min}$ to remove solids.

DEHP has been shown to be more soluble in acetonitrile than in non-polar fats in the cheese matrix (Castle et al. 1990). Accordingly, each hexane extract was partitioned into $3.3 \mathrm{~L}$ of hexane-saturated acetonitrile, the acetonitrile phase separated, evaporated to $2 \mathrm{~L}$, and stored at $-20^{\circ} \mathrm{C}$. After chilling, the acetonitrile extract was gravity-filtered while still cold to remove protein and fat precipitates. The DEHP in these extracts and contemporaneously processed blanks was further purified using a series of 3 chromatographic separations: silica-gel "flash" chromatography (FC); size exclusion chromatography (SEC); and reverse-phase high-performance liquid chromatography (HPLC). Isolation of DEHP required approximately 1 pass through the FC column, 1 through the SEC column, and 4 passes through the HPLC column, per $400 \mathrm{~g}$ of cheese. Thus, for a typical batch of cheese averaging $1.6 \mathrm{~kg}, 4,4$, and 16 column passes were required, respectively.

\section{Liquid Chromatography Separations}

Each 2-L acetonitrile sample was split into seven $285-\mathrm{mL}$ portions that were rotary-evaporated and then purified on 7 separate precleaned FC columns using $175 \mathrm{~g}$ of 32-63 $\mu \mathrm{m}$ "flash"-grade silica gel particles (Dynamic Adsorbents, Atlanta, GA, USA). The DEHP fractions were typically collected in $300 \mathrm{~mL}$ of a $1.6 \%$ acetone: $98.4 \%$ hexane (volume fraction) mobile phase after elution with $500 \mathrm{~mL}$ of $100 \%$ hexanes and $1100 \mathrm{~mL}$ of the $1.6 \%$ acetone solution, as determined by gas chromatography electron-impact-mass spectrometry (GC-EIMS).

Each of the 7 DEHP-containing fractions were subsequently rotary-evaporated to $1 \mathrm{~mL}$ and further purified by SEC using tandem 30-cm, 21-mm-ID Oligopore columns (Agilent Technologies, Palo Alto, CA, USA) containing 6- $\mu \mathrm{m}$ particles. DEHP was eluted with $100 \%$ methylene chloride and collected in $10-\mathrm{mL}$ fractions. The 7 DEHP fractions were combined, desolvated by rotary evaporation, and reconstituted in $1.5 \mathrm{~mL}$ of acetonitrile.

The final purification was achieved with a $15-\mathrm{cm} \times 9.4-\mathrm{mm}-\mathrm{ID}$ C18 column (Agilent Zorbax Eclipse), by eluting with a $95 \%$ acetonitrile: $5 \%$ water mixture at $4 \mathrm{~mL} / \mathrm{min}$ for $15 \mathrm{~min}$ after elution with $90 \%$ acetonitrile: $10 \%$ water mobile phase for $20 \mathrm{~min}$. DEHP was observed by UV absorption 


\section{MA Nelson et al.}

at $254 \mathrm{~nm}$ and collected in $40-\mathrm{mL}$ borosilicate glass vials with PTFE-lined caps after $33 \mathrm{~min}$. Each sample yielded $80 \mathrm{~mL}$ of HPLC eluent from which solvent was removed by rotary evaporation in a $10-\mathrm{mL}$ pear-shaped recovery flask and reconstituted in $1 \mathrm{~mL}$ of methylene chloride.

Table 1 Sample and concurrent blank processing.

\begin{tabular}{|c|c|c|c|c|c|c|c|}
\hline Process element & Batch 1 & Batch 2 & Batch $3^{a}$ & Batch 4 & Batch 5 & Batch 6 & Batch $7^{b}$ \\
\hline Cheese wheel ID & 1 & 1 & 2 & 2 & 2 & 2 & 2 \\
\hline Extraction date & $8 / 24 / 2011$ & $9 / 26 / 2011$ & $2 / 14 / 2012$ & $2 / 27 / 2012$ & $2 / 27 / 2012$ & $4 / 3 / 2012$ & $4 / 3 / 2012$ \\
\hline $\begin{array}{l}\text { Mass of cheese } \\
\text { extracted (g) }\end{array}$ & 2640 & 2622 & 1192 & 1633 & 1687 & 1247 & 1262 \\
\hline $\begin{array}{l}\text { Contemporaneously } \\
\text { processed blank }\end{array}$ & 1 & - & 2 & 3 & 3 & 4 & 4 \\
\hline $\begin{array}{l}\text { Number of column } \\
\text { passes, isolate/MBlk }\end{array}$ & $35 / 35$ & $35 / \mathrm{na}^{\mathrm{b}}$ & $19 / 21$ & $23 / 12$ & $26 / 12$ & $19 / 20$ & $21 / 20$ \\
\hline
\end{tabular}

a Some of batch isolate lost.

${ }^{b}$ na $=$ blank data not applicable for this batch.

To minimize contamination, glassware, utensils, and aluminum foil coverings were washed, baked at $250{ }^{\circ} \mathrm{C}$ for $12 \mathrm{hr}$, and rinsed with acetone. Sample handling, extraction, and purification methods were performed using glass containers and metallic utensils. LC columns were thoroughly rinsed with the mobile phase that most strongly eluted any stationary phase-retained contaminant DEHP prior to each individual separation.

\section{Analyses}

Post-HPLC samples and blanks were analyzed for DEHP and co-eluting compounds by GC-EIMS using an Agilent Technologies (Wilmington, DE, USA) 6890N Network GC system, with a 7683 Series Autosampler, and 5973 inert quadrupole mass-selective detector. Analyses were performed with $1.0-\mu \mathrm{L}$, on-column injections to a $0.25-\mathrm{mm} \times 60-\mathrm{m}$, DB-XLB (Agilent) polysiloxane wallcoated capillary column preceded by a $5-\mathrm{m}$ deactivated fused-silica capillary retention gap, with a $1.3 \mathrm{~mL} / \mathrm{min}$ helium flow, at an injection temperature of $83{ }^{\circ} \mathrm{C}$. The $\mathrm{GC}$ was temperature programmed (hold $60^{\circ} \mathrm{C}$ for $3 \mathrm{~min}$; ramp $45^{\circ} \mathrm{C} / \mathrm{min}$ to $200^{\circ} \mathrm{C}$; followed by $7.5^{\circ} \mathrm{C} / \mathrm{min}$ to $320^{\circ} \mathrm{C}$; hold $3 \mathrm{~min}$ ) to elute DEHP at $21 \mathrm{~min}$. The solvent (in each case methylene chloride) was eluted prior to $6 \mathrm{~min}$ and, in preliminary analyses, no other compounds could be observed between the solvent elution time and $6 \mathrm{~min}$; and none could be observed between 26 and 76 min after sample injection. Therefore, spectra were collected between 6 and $26 \mathrm{~min}$. Mass scans from 50 to $300 \mathrm{amu}(\mathrm{m} / \mathrm{z})$ were acquired every $0.006 \mathrm{~min}$ and the entire $251-\mathrm{m} / \mathrm{z} \times 3468$-scan matrix was stored for processing. This program and instrument were used for all GC-EIMS analyses to minimize differences in retention times and column-bleed background between subsequent $\mathrm{m} / \mathrm{z}$ spectra and total ion chromatograms. Three 6-point analytical calibration curves were made with petrogenic DEHP $(99.8 \pm 0.1 \%$ pure; Supelco Analytical, Bellefonte, PA, USA) in each of 2 ranges (70-86 and $0.4-1.5 \mu \mathrm{g} / \mathrm{g}$ in methylene chloride) such that they tightly bracketed the concentration of DEHP in all determinations. Uncertainty of DEHP determinations was determined to be $\leq 2 \%$ in both calibration ranges (M Nelson, unpublished data). Results of these DEHP determinations are listed in Table 2. In addition to DEHP, small amounts of di-n-butyl phthalate, and 28 fatty acids and fatty acid esters were identified in each isolate from its fragmentation ion mass spectra. Each of the latter accounted for $0.04 \%$ to $4.10 \%$ of the counts in the total ion chromatogram (TIC). Di-n-butyl phthalate accounted for an additional $0.05 \pm 0.01 \%$ of TIC counts. 
Contemporary Fraction of bis(2-ethylhexyl) in Stilton Cheese

Table 2 Analytical results.

\begin{tabular}{|c|c|c|c|c|c|c|}
\hline Sample ID & $\begin{array}{l}\text { Mass C as } \\
\operatorname{DEHP}^{a}(\mu \mathrm{g})\end{array}$ & $\begin{array}{l}\text { Purity } \\
(\%)\end{array}$ & $\begin{array}{l}\text { Mass } C^{c} \\
(\mu g)\end{array}$ & $\begin{array}{l}\mathrm{GCMS}^{\mathrm{d}} \\
(\mu \mathrm{g})\end{array}$ & $\begin{array}{l}\mathrm{F}^{14} \mathrm{C}^{\mathrm{e}} \\
\left(\mathrm{Fm}_{\text {reported }}\right)\end{array}$ & $\begin{array}{l}\delta^{13} \mathrm{C} \\
\mathrm{VPDB}^{\mathrm{f}}\end{array}$ \\
\hline \multicolumn{7}{|c|}{ Measured sample carbon } \\
\hline \multicolumn{7}{|l|}{ Isolates } \\
\hline STO1 & $92.18 \pm 4.65$ & $91.2 \pm 2.7$ & $109 \pm 3$ & $100.4 \pm 5.9$ & $0.284 \pm 0.004$ & $-30.0 \pm 0.04$ \\
\hline STO2 & $67.25 \pm 0.76$ & $88.1 \pm 1.7$ & $99 \pm 3$ & $76.7 \pm 1.5$ & $0.281 \pm 0.004$ & $-29.3 \pm 0.3$ \\
\hline STO3 & $63.41 \pm 0.79$ & $87.2 \pm 1.7$ & $127 \pm 2$ & $72.5 \pm 1.5$ & $0.353 \pm 0.003$ & $-29.3 \pm 0.3$ \\
\hline STO4 & $65.42 \pm 0.76$ & $90.5 \pm 1.7$ & $135 \pm 2$ & $72.1 \pm 1.3$ & $0.311 \pm 0.003$ & $-29.3 \pm 0.3$ \\
\hline STO5 & $69.09 \pm 0.84$ & $92.3 \pm 1.4$ & $114 \pm 2$ & $75.1 \pm 1.3$ & $0.334 \pm 0.003$ & $-29.3 \pm 0.3$ \\
\hline \multicolumn{7}{|c|}{ DEHP standards } \\
\hline STD1 & $147.5 \pm 1$ & $99.8 \pm 0.1^{\mathrm{g}}$ & $135 \pm 2$ & & $0.0018 \pm 0.0042$ & $-29.3 \pm 0.16$ \\
\hline STD2 & $143 \pm 1$ & $99.8 \pm 0.1^{\mathrm{g}}$ & $130 \pm 2$ & & $0.0000 \pm 0.0044$ & $-29.3 \pm 0.16$ \\
\hline \multicolumn{7}{|c|}{ Whole cheese } \\
\hline & - & - & - & - & $1.045 \pm 0.002^{\mathrm{g}}$ & $-27.1 \pm 0.03^{\mathrm{g}}$ \\
\hline
\end{tabular}

Blanks - DEHP "pure" spike and spiked-method blanks

\begin{tabular}{lllrlll} 
& & \multicolumn{5}{c}{$\begin{array}{l}\text { Mass C of } \\
\text { spike }(\mu \mathrm{g})\end{array}$} \\
S1 & $47.32 \pm 0.04$ & $99.8 \pm 0.1^{\mathrm{h}}$ & $78 \pm 2$ & $47.32 \pm 0.04$ & $0.011 \pm 0.005$ & $-29.3 \pm 0.16$ \\
S2 & $47.98 \pm 0.04$ & $99.8 \pm 0.1^{\mathrm{h}}$ & $81 \pm 2$ & $47.98 \pm 0.04$ & $0.008 \pm 0.005$ & $-29.3 \pm 0.16$ \\
S3 & $47.75 \pm 0.04$ & $99.8 \pm 0.1^{\mathrm{h}}$ & $83 \pm 2$ & $47.78 \pm 0.04$ & $0.008 \pm 0.005$ & $-29.3 \pm 0.16$ \\
SB1k1 & $46.05 \pm 0.06$ & $\mathrm{ND}^{\mathrm{i}}$ & $68 \pm 2$ & $45.39 \pm 0.04$ & $0.037 \pm 0.005$ & $\mathrm{ND}$ \\
SB1k2 & $48.04 \pm 0.04$ & ND & $88 \pm 2$ & $47.85 \pm 0.04$ & $0.022 \pm 0.005$ & ND \\
SB1k3 & $47.87 \pm 0.04$ & ND & $78 \pm 2$ & $45.59 \pm 0.04$ & $0.064 \pm 0.005$ & ND \\
\hline
\end{tabular}

${ }^{a}$ Determined by analytical GCMS analyses, except for gravimetrically prepared aliquots of the Supelco standard DEHP; $1 \sigma$ combined uncertainty; $n=3$.

${ }^{b}$ Determined by deconvolution of GC-EIMS data; uncertainty at $95 \%$ confidence interval; $n=5$.

${ }^{c}$ Determined by $\mathrm{CO}_{2}$ pressure-volume manometry after combustion; $1 \sigma$ uncertainty; $n=1$.

${ }^{\mathrm{d}}$ Determined by analytical GCMS analyses and purity determinations; $1 \sigma$ combined uncertainty, $n=1$.

${ }^{\mathrm{e}}$ Modern fraction of carbon in AMS-analyzed sample, corrected for combustion blank of $1 \mu \mathrm{g} \mathrm{C}\left(F m_{\text {reported }}\right)$; $2 \sigma$ uncertainty; $n=1$.

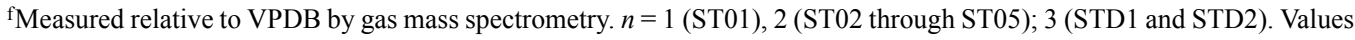
used for S1, S2, and S3 were that of the mean of STD1 and STD2; $1 \sigma$ uncertainty.

g $1 \sigma$ uncertainty; $n=3$.

${ }^{\mathrm{h}}$ Average and $1 \sigma$ uncertainty of HPLC-UV and GC-FID determinations; $n=2$.

${ }^{\mathrm{i}} \mathrm{ND}=$ not determined.

To permit corrections to be made for isotope fractionation during AMS, chromatographic steps, and biological processes, values of $\delta^{13} \mathrm{C}$ with respect to Pee Dee belemnite (VDPB) were determined by ${ }^{13} \mathrm{C} /{ }^{12} \mathrm{C}$ mass spectrometry (Isoprime, Ltd, Manchester, UK). Three 10 - to $15-\mu \mathrm{g}$ quantities of DEHP isolate from cheese (2 separate samples from wheel 1 and 1 aggregate isolate from wheel 2), three 72 - to $100-\mu \mathrm{g}$ quantities of lyophilized whole Stilton cheese, and three 30- to $61-\mu \mathrm{g}$ quantities of the Supelco petrogenic DEHP standards were analyzed.

All samples to be analyzed by AMS were reduced to $200 \mu \mathrm{L}$ in $95 \%$ n-hexane and sent from the University of Maryland College Park to LLNL CAMS in borosilicate vials with PTFE-lined caps. These included the 5 DEHP isolates (ST01 through ST05), 3 DEHP-spiked method blanks (SBlk1, SBlk2, and SBlk3), 3 "pure" spikes (S1, S2, and S3), and the ( $n=2)$ Supelco petrogenic DEHP standards (STDn) listed in Table 2. Note that both the pure DEHP spikes and standards were submitted without processing except for dilution in hexane and transfer to the shipping vials prior to shipping to LLNL. 


\section{A Nelson et al.}

At LLNL, samples were transferred to precombusted $\left(900{ }^{\circ} \mathrm{C}\right.$ for $3.5 \mathrm{hr}$ ) quartz combustion tubes from which solvent was evaporated overnight at $50{ }^{\circ} \mathrm{C}$. Excess $\mathrm{CuO}$ was added to each quartz tube. Tubes were evacuated with an oil-free turbopump station, sealed with a $\mathrm{H}_{2} / \mathrm{O}_{2}$ torch, and heated at $900{ }^{\circ} \mathrm{C}$ for $3.5 \mathrm{hr}$ to oxidize all carbon to $\mathrm{CO}_{2}$. The $\mathrm{CO}_{2}$ was cryogenically isolated from other combustion products and its carbon mass manometrically determined with a precision of $1.5 \%$ to $3.0 \%$, followed by graphitization with iron catalyst in individual reactors (Vogel et al. 1987; Santos et al. 2004). All ${ }^{14} \mathrm{C} /{ }^{12} \mathrm{C}$ measurements were made on the graphitized samples at LLNL CAMS on the 10MV High Voltage Engineering Europa (HVEE) FN-class tandem electrostatic AMS system. Corrections for background contamination introduced during sample preparation were made following standard procedures (Brown and Southon 1997). All data were normalized to the mean of 6 identically prepared NIST SRM 4990B (oxalic acid I) standards. NIST SRM 4990C, IAEA C-6, and TIRI wood served as quality control secondary standards to monitor spectrometer performance. ${ }^{14} \mathrm{C} / \mathrm{C}$ concentrations are reported using the $\mathrm{F}^{14} \mathrm{C}$ (fraction modern) nomenclature for reporting post-bomb data defined in Equation 2 of Reimer et al. (2004). The measurement error was determined for each sample and is reported as $1 \sigma$.

\section{Estimation of Isolate Purity}

Purity is often determined from the areas of the compounds detected in the chromatogram. To assure that no additional compounds were eluting under the DEHP peak, the DEHP was removed from the GC-EIMS matrix for each isolate by non-negative least-squares deconvolution with that of a Supelco high-purity standard of similar concentration, after reducing the number of time-scans 6fold by binning to minimize the effects of small retention time variations. Ions detected during the period of DEHP elution were highly correlated and consequently the residual in this region was small (i.e. $0.04 \%$ of the counts obtained in the total sample spectrum) and contained no discernible co-eluting peaks. The total residual was free of GC column bleed across the entire chromatogram. The relative masses of co-eluting compounds and DEHP were estimated for each mass fragmentation channel in the residual and isolate spectra. Subsequently, the mass fractions of carbon in the isolate and residual matrices $(0.743 \pm 0.001$ and $0.781 \pm 0.002$, respectively) were determined as TICpeak-area-weighted means of the carbon mass fractions of the identified compounds. The purity of carbon from DEHP in each sample was then estimated from the amount of co-eluted carbon determined in the residual, $\left(N_{C}\right)_{\text {residual }}$, and isolate matrices, $\left(N_{C}\right)_{\text {isolate }}$, i.e.

$$
\text { Purity }_{D E H P}=1-\frac{\left(N_{C}\right)_{\text {residual }}}{\left(N_{C}\right)_{\text {isolate }}}
$$

The calculated purities varied with binning, and consequently their uncertainties were estimated as the standard deviation of results obtained for 5 binning factors $(3,4,6,12$, and 17). As indicated in Table 2, DEHP purity of the isolates ranged from $88.1 \pm 1.4 \%$ to $92.3 \pm 1.1 \%$.

\section{DATA ANALYSIS}

\section{Processing Laboratory Carbon Blanks}

In this work, 3 components to the total processing laboratory carbon blank (TBlk) were identified: 1) carbon in the form of DEHP measured in the method blanks; 2) carbon in the co-eluted compounds; and 3) extraneous carbon. They were determined as described below and results are listed in Table 3.

Owing to the differences between batch sizes and collection periods for samples and method blanks, carbon in the 3 method blanks had to be apportioned to represent its contribution to carbon in each 
of the 5 isolates. Only DEHP was detected in the method blanks. Its carbon mass contribution to each isolate $\left(m_{C_{\text {DEHPMBIK }}}\right)$ was assumed to scale with the number of LC column passes of the sample relative to that of the blank or blanks to which it was assigned. Since all samples, except for ST01 and ST05, were made by combining portions of isolates from 2 batches and were also associated with 2 different blanks, their contributions from each blank were scaled separately and summed. Isolate ST01 was associated with its own (scaled) method blank value and, for ST05, the method blank value was the scaled average of the DEHP concentration in the 4 method blanks.

The mass of co-eluted carbon, $m_{C_{c o e l}}$, in each isolate was calculated directly from the isolate's DEHP carbon mass, $\left(m_{C_{D E H P}}\right)_{\text {isolate }}$, and its purity, $\left(\text { Purity }_{D E H P}\right)_{\text {isolate }}$. This is

$$
m_{C_{\text {coel }}}=\left(\frac{m_{C_{D E H P, \text { iso }}}}{\text { Purity }}\right)\left(1-\text { Purity }_{D E H P, \text { iso }} \text { iso }\right)
$$

Although DEHP and co-eluted compound carbon blanks could be determined by GC-EIMS, it was evident from differences between manometrically determined $\mathrm{C}$ mass (after sample combustion at CAMS; column 4 in Table 2) and our estimates of C mass from analytical GC-EIMS (column 5), that the samples contained substantial amounts of extraneous carbon. This extraneous carbon $\left(m_{C_{T e x t r}}\right)$ is attributed to either non-detectable compounds (e.g. polymeric materials) or particles (e.g. detritus, dust, and soot) that deposited in open apparatus (such as flash-chromatography columns and fraction collection containers), or both. The total mass of $\mathrm{C}$ in the method blank $\left(m_{C T_{B l k}}\right)$ is simply the sum of the 3 other components listed in Table 3.

Table 3 Masses of components of total method blank.

\begin{tabular}{|c|c|c|c|c|}
\hline Sample ID & $\begin{array}{l}m_{C_{D E H P M B l k}} \\
(\mu \mathrm{g} \mathrm{C})^{\mathrm{a}}\end{array}$ & $\begin{array}{l}m_{C_{c o e l}} \\
(\mu \mathrm{g} \mathrm{C})^{\mathrm{b}}\end{array}$ & $\begin{array}{l}m_{C_{\text {Textr }}} \\
(\mu \mathrm{g} \mathrm{C})^{\mathrm{c}}\end{array}$ & $\begin{array}{l}m_{C T_{B l k}} \\
(\mu \mathrm{g} \mathrm{C})^{\mathrm{c}}\end{array}$ \\
\hline STO1 & $0.11 \pm 0.03$ & $7.47 \pm 0.44$ & $8.64 \pm 6.14$ & $16.22 \pm 6.16$ \\
\hline STO2 & $1.12 \pm 0.04$ & $9.49 \pm 0.18$ & $22.57 \pm 2.69$ & $33.18 \pm 2.70$ \\
\hline STO3 & $0.89 \pm 0.07$ & $9.05 \pm 0.18$ & $54.28 \pm 2.58$ & $64.22 \pm 2.59$ \\
\hline STO4 & $0.76 \pm 0.06$ & $6.67 \pm 0.12$ & $62.63 \pm 2.49$ & $70.06 \pm 2.49$ \\
\hline STO5 & $0.92 \pm 0.06$ & $5.97 \pm 0.10$ & $38.73 \pm 2.49$ & $45.62 \pm 2.49$ \\
\hline
\end{tabular}

\section{Laboratory Blank Corrections to Fm}

The values of $\mathrm{F}^{14} \mathrm{C}$, denoted as $F m_{\text {reported }}$ in the following calculations, are listed in Table 2 and are linear combinations of the modern fraction of the DEHP carbon in each isolate $\left(F m_{D E H P, \text { iso }}\right)$, and that of the total laboratory carbon blank $\left(F m_{T B l k}\right)$. Thus,

$$
F m_{\text {reported }}=F m_{D E H P, \text { iso }} \cdot \gamma_{D E H P}+F m_{T b l k} \cdot \gamma_{T b l k}
$$

where $\gamma_{D E H P}$ and $\gamma_{T B l k}$ are the mass mixing ratios of the DEHP carbon in the isolate and the total laboratory blank, respectively. Noting that $\gamma_{D E H P}=\left(1-\gamma_{T B l k}\right)$, the laboratory blank-corrected values of the modern carbon fractions of the DEHP $\left(F m^{\prime}\right)$ in the isolates were calculated from data in Tables 2 and 3 as follows: 


\section{MA Nelson et al.}

$$
F m_{\text {isolate }}{ }^{\prime}=\frac{F m_{\text {reported }}-F m_{T B l k} \cdot \gamma_{T B l k}}{1-\gamma_{T B l k}}
$$

where

$$
\gamma_{T B l k}=\frac{m_{C T_{B l k}}}{m_{C T_{B l k}}+m_{C_{D E H P, \text { iso }}}}
$$

In Equation 4, $F m_{T B l k}$ for each isolate was calculated from the linear combination of its 3 laboratory blank components, i.e.

$$
F m_{T B l k}=\gamma_{D E H P_{M B l k}} \cdot F m_{D E H P_{M B l k}}+\gamma_{C o e l} \cdot F m_{C o e l}+\gamma_{\text {extr }} \cdot F m_{\text {extr }}
$$

where $\gamma \mathrm{s}$ are mass mixing coefficients defined as follows:

$$
\gamma_{D E H P_{M B l k}}=\frac{m_{C_{D E H P M B l k}}}{m_{C_{T B l k}}}, \gamma_{D E H P_{M B l k}}=\frac{m_{C_{C o e l}}}{m_{C_{T B l k}}} \text {, and } \gamma_{e x t r}=\frac{m_{C_{e x t r}}}{m_{C_{T B l k}}}
$$

each of which was calculated with data in Table 3. Method blanks were deemed to contain too little mass for AMS and so the values of $F m_{D E H P_{M B l k}}$ were assumed to be the mean of values $(0.0009)$ reported for the petrogenic Supelco DEHP standard (Table 2). As the co-eluted mass consisted of natural fatty acids and fatty acid esters from the cheese matrix, $\mathrm{Fm}_{\mathrm{Coel}}$ was assigned to that of the lyophilized whole cheese $(F m=1.045 \pm 0.002)$. The modern fraction of extraneous carbon in Equation 5, $\mathrm{Fm}_{\text {extr }}$, was determined from the spiked-blank data.

\section{Determination of $\mathbf{F m}$ values for Extraneous Carbon}

As can be determined from Table 2, the $F m$ values reported for the pure spikes $(0.009 \pm 0.001$, average and standard error of the mean for pure spikes S1, S2, and S3) were uniformly 10-fold greater than those $(0.0009 \pm 0.003)$ reported for the much larger DEHP standard. Thus, it was clear that the "pure" spikes contained some extraneous modern carbon mass and that it was accrued during postGC-EIMS operations (dilution, packaging, and shipping). Herein, this post-GC-EIMS component of

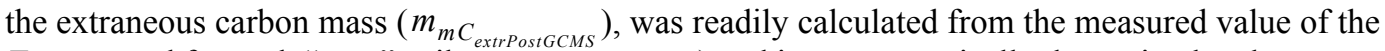
$F m$ reported for each "pure" spike $F m_{\text {reported }_{\text {spike }} S}$ ) and its manometrically determined carbon mass $\left(m_{C_{\mathrm{CO}_{2} \text {, spikes }}}\right)$ as follows:

$$
m_{m C_{\text {extrPost GCMS }}}=m_{C_{C_{2}, \text { spikes }}} \cdot F m_{\text {reported }}{ }_{\text {spike }} S-m_{C_{\text {spikeSDEHP }}} \cdot F m_{\text {spikeS }}
$$

where the product $m_{C_{\text {spikesDEHP }}} \cdot F m_{\text {spikes }}$ is the mass of modern carbon in the DEHP used to prepare each "pure" spike (S1, S2, or S3) and $F m_{\text {spikes }}$ is again, that of the Supelco standard, i.e. $0.0009 \pm$ 0.0009 . The average $m_{m C_{\text {extr PostGCMS }}}$ was $0.725 \pm 0.12 \mu \mathrm{g} \mathrm{C}$ and was assumed to be applicable to all isolates, except ST01.

The remaining modern extraneous carbon is clearly attributed to pre-GC-MS processing (i.e. $m_{\left.m C_{\text {extrPreGCMS }}\right)}$ and was determined analogously for each of the spiked method blanks from its mass $\left(m_{m C_{C_{2} S B l k}}\right)$ and measured modern fraction $\left(\right.$ Fm $\left._{\text {reported }_{S B l k}}\right)$ as follows:

$$
m_{m C_{\text {extrPreGCMS }}}=m_{m C_{C_{2} O_{B B k}}} \cdot F m_{\text {reported }}{ }_{S B l k}-m_{C_{S B k D E H P}} \cdot 0.0009-0.725 \mu \mathrm{g}
$$


Values of $m_{m C_{\text {extrPreGCMS }}}$ calculated in this way were apportioned to each respective isolate as outlined above for the apportioning of $m_{C_{D E H P M B I}}$. The apportioned values are listed in Table 3 . The total extraneous modern carbon masses, and pre- and post-GCMS attributed to each isolate $\left(m_{m C_{\text {Textrisolate }}}\right)$ are listed in Table 4. Finally, the values of $F m_{\text {extr }}$ needed to calculate $F m_{T B l k}$ for each isolate using Equation 5 were determined using the definition

$$
F m_{\text {extr }}=\frac{m_{m C_{\text {Textrisolate }}}}{m_{C_{\text {Textr }}}}
$$

and these are also listed in Table 4.

Table 4 Reported and component blank fractions of modern carbon values used to determine $F c_{D E H P_{\text {cheese }}}$ of each isolate.

\begin{tabular}{lllll}
\hline Sample ID & $F m_{\text {reported, } i s o}{ }^{\mathrm{a}}$ & $F m_{\text {extr }}{ }^{\mathrm{b}}$ & $F m_{\text {TBlk }}{ }^{\mathrm{c}}$ & $F c_{D E H P_{\text {cheese }}}{ }^{\mathrm{d}}$ \\
\hline ST01 & $0.284 \pm 0.004$ & $0.190 \pm 0.090$ & $0.580 \pm 0.497$ & $0.213 \pm 0.032$ \\
ST02 & $0.281 \pm 0.004$ & $0.435 \pm 0.068$ & $0.593 \pm 0.117$ & $0.128 \pm 0.040$ \\
ST03 & $0.353 \pm 0.003$ & $0.220 \pm 0.023$ & $0.333 \pm 0.039$ & $0.302 \pm 0.044$ \\
ST04 & $0.311 \pm 0.003$ & $0.208 \pm 0.012$ & $0.285 \pm 0.029$ & $0.277 \pm 0.042$ \\
ST05 & $0.334 \pm 0.003$ & $0.255 \pm 0.098$ & $0.353 \pm 0.137$ & $0.253 \pm 0.037$ \\
\hline
\end{tabular}

a $2 \sigma$ uncertainty; $n=1$.

b $1 \sigma$ combined uncertainty; $n=1$.

c $1 \sigma$ combined uncertainty; $n=1$.

${ }^{\mathrm{d}} 1 \sigma$ Monte Carlo-derived uncertainty; $n=100,000$.

\section{Fractionation Corrections and Contemporary Carbon Calculation}

For the purpose of this study, the $F m$ values reported in Table 2 were converted to their corresponding fractions of "contemporary" carbon, $F C$, determined with respect to the value of $F m$ of the lyophilized whole cheese matrix and corrected for ${ }^{14} \mathrm{C}$ fractionation as per Stuiver and Polach (1977) as shown in Equation 6. Use of whole cheese as a contemporary reference standard eliminates the need to correct for differences in ${ }^{14} \mathrm{C}$ fractionation between the cheese and the oxalic acid standard, as well as for fluctuations in atmospheric ${ }^{14} \mathrm{C}$ since 1950 due to nuclear weapons testing.

$$
F C_{D E H P_{\text {cheese }}}=\left(\frac{F m_{\text {isolate }}}{F m_{\text {wholecheese }}}\right)\left[\frac{\left(1-2 \frac{\left(\delta^{13} C_{V P D B}\right)_{\text {isolate }}}{1000}\right)}{\left(1-2 \frac{\left(\delta^{13} C_{V P D B}\right)_{\text {wholecheese }}}{1000}\right)}\right]
$$

In this equation, $F C_{D E H P_{\text {cheese }}}$ is the fraction of contemporary carbon from DEHP in the isolate after all corrections, and thus that in the cheese sample, and $F m_{\text {wholecheese }}$ is the fraction of modern carbon reported for the lyophilized whole Stilton cheese $(1.04 \pm 0.008)$. Respective $\delta^{13} \mathrm{C}(\mathrm{VPDB})$ are listed in Table 2. The fraction of contemporary carbon in isolates from Stilton cheese, $F m_{\text {reported,iso }}$, along with their respective $\mathrm{Fm}_{\text {extr }}, \mathrm{Fm}_{T B l k}$, and calculated fractions of contemporary carbon, $F C$, of DEHP are presented in Table 4. 


\section{MA Nelson et al.}

\section{Uncertainty Analysis}

Uncertainties in $F C_{D E H P}$ values reported herein (Table 4) were estimated using the Monte Carlo method as follows. Equation 6 and supporting Equations (3-5) for calculation of derived

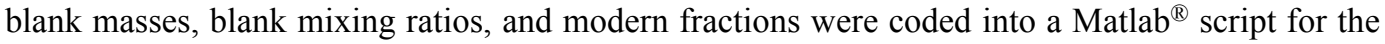
calculation of $F C_{D E H P_{\text {cheese }}}$ of each isolate. Specifically, blank carbon masses $\left(m_{C_{c o e l}}, m_{C_{\text {Textr }}}\right.$, $\left.m_{C_{T B l k}}, m_{m C_{\text {extrPostGCMS }}}, m_{m C_{\text {extrPreGCMS }}}\right)$, mass mixing ratios $\left(\gamma_{T B l k}, \gamma_{D E H P_{M B l k}}, \gamma_{D E H P_{M B l k}}\right.$, and $\left.\gamma_{\text {extr }}\right)$, and blank modern fractions $\left(F m_{T B l k}\right.$ and $\left.F m_{\text {extr }}\right)$ were calculated as per the equations and relationships described above. Values of $m_{C_{D E H P M B l k}}, m_{C_{D E H P, \text { iso }}}$, PVM carbon mass, isolate purity, the remaining modern fractions $\left(F m_{C o e l}, F m_{D E H P_{M B l k}}, F m_{\text {reported }}\right.$, and $\left.F m_{\text {wholecheese }}\right)$, and $\delta^{13} C_{V P D B}$ were input as received from the measurement laboratories. We note that $F m_{\text {reported }}, \mathrm{CO}_{2}$ manometrically derived (PVM) carbon masses, and $\delta^{13} C_{V P D B}$ were input for all sample IDs listed in Table 2, i.e. including those for the spikes and spiked blanks. These input variables, totaling 16, are listed in Table 5 for isolates, spikes, and spiked blanks. Subsequently, for each of the 16 variables, the Matlab function, randn, was used to populate a matrix of 100,000 inputs randomly chosen from a normal distribution of values centered at the mean of each variable and having a standard deviation corresponding to the uncertainty in each. Uncertainties in all entered variables were estimates of $1 \sigma$, except for $F m_{D E H P}$ and $F m_{\text {reported }}$ where $2 \sigma$ uncertainties were used. In the latter, individual measurement uncertainty exceeded the standard deviation estimate of the 2 values and so a $2 \sigma$ uncertainty of the mean was used.

Table 5 Example input parameters used with Monte Carlo analysis determination of $F C_{D E H P_{\text {cheese }}}$ and uncertainty assessment.

\begin{tabular}{llll}
\hline Parameter & Description of parameter & Sample ST05 & SBlk1 \\
\hline PVM & Mass of C by C0 & $114 \pm 2$ & $68 \pm 2$ \\
$m_{C_{D E H P, \text { iso }}}$ & Mass of C as isolated DEHP & $69.09 \pm 0.84$ & $0.68 \pm 0.05$ \\
$m_{C_{\text {spikeSDEHP }}}$ & Mass of C in DEHP spike & - & $45.39 \pm 0.04$ \\
$m_{C_{D E H P M B l k}}$ & Mass C DEHP in apport. blank & $0.92 \pm 0.06$ & - \\
$F m_{\text {Coel }}$ & Modern fraction co-eluted & $1.045 \pm 0.002$ & - \\
$F m_{D E H P}$ & Modern fraction fossil DEHP & $0.0009 \pm 0.009$ & $0.0009 \pm 0.009$ \\
Purity & By deconvolution & $0.918 \pm 0.014$ & - \\
$F m_{\text {reported }}$ & Fraction modern of sample & $0.3335 \pm 0.0031$ & $0.037 \pm 0.005$ \\
$F m_{\text {wholecheese }}$ & Fraction modern of whole cheese & $1.0405 \pm 0.0081$ & - \\
$\left(\delta^{13} C_{V P D B}\right)_{\text {isolate }}$ & $\delta^{13}$ C fractionation for the isolate & $-29.3 \pm 0.3$ & - \\
$\left(\delta^{13} C_{V P D B}\right)_{\text {wholecheese }}$ & $\delta^{13} \mathrm{C}$ fractionation for the whole & $-27.1 \pm 0.03$ & - \\
$\left(\delta^{13} C_{V P D B}\right)_{D E H P}$ & cheese & & $-29.1 \pm 0.04$ \\
\hline
\end{tabular}

\section{RESULTS AND DISCUSSION}

As indicated in Table 4, FC of DEHP from cheese ranged from a low of $0.128 \pm 0.040$ (ST02) to $0.302 \pm 0.044$ (ST03) and the mean of Monte Carlo-perturbed values calculated for all isolates was $0.235 \pm 0.073(1 \sigma$, and \pm 0.091 at $95 \%$ confidence interval $)$, indicating that most of the DEHP was petrogenic. The value for ST02 appears inordinately low. However, applying the Grubbs (1969) test, we find that its difference from the Monte Carlo mean is not significant at the $95 \%$ confidence level and, thus, is not a statistical outlier. This value could be the result of an inhomogeneity of DEHP in the cheese. Not surprisingly, we find from sensitivity calculations, that $F C$ values are sensitive to their corresponding $m_{C_{T e x t r}}$ values, and consequently increase with increasing carbon mass values 
determined from $\mathrm{CO}_{2}$ measurements after combustion, from which the latter are calculated. If the manometrically derived value for ST02 were $139.4 \mu \mathrm{g}$, i.e. representative of a $63-\mu \mathrm{g}$ extraneous carbon mass (and more comparable to spiked blank SBlk1), instead of $22.3 \mu \mathrm{g}$, then its $F C$ would be $0.291 \pm 0.042$. Moreover, we note that $F m$ values are independent of the carbon mass (so long as the mass is homogenous) and that there was little difference in the $\mathrm{F}^{14} \mathrm{C}$ values reported for ST02 $(0.281 \pm 0.004)$ and the mean $(0.333 \pm 0.021)$ for ST03, ST04, and ST05. We suspect, therefore, that the entire sample was not combusted to $\mathrm{CO}_{2}$, possibly owing to loss during transport. The difference between $139.4 \mu \mathrm{g}$ and the manometrically derived value of $99 \mu \mathrm{g}$ used in the analysis above is equivalent to the loss of $50 \mu \mathrm{L}$ of the sample in solution prior to combustion.

As noted above, the total carbon blank, averaging $45 \mu \mathrm{g}$, was determined to be substantial. About $17 \%$ of this was co-eluted carbon derived from the cheese matrix and therefore expected. The remaining amount represents extraneous carbon, of which from analysis of the pure spikes, $30 \mu \mathrm{g}$ (i.e. $\sim 80 \%$ ), could only be associated with the shipping vials. As the caps could not be baked, they are likely the source of this contamination. In subsequent tests, hexadecamethylheptasiloxane, a component of the silicone septum affixed to the top of the Teflon ${ }^{\mathrm{TM}}$ liner, could be readily detected by GC-EIMS after pure hexane was shaken in a clean vial that had been successively uncapped and recapped as required to switch from the acetonitrile-water HPLC eluent to hexane and to perform concomitant rotary evaporations. It is notable that this compound was not detectable when the vial was capped and reopened only once. However, the vial caps had been washed with a dilute solution of Alconox ${ }^{\mathrm{TM}}$, for which a preliminary $F m_{\text {reported }}$ was subsequently determined by AMS to be $0.28 \pm$ 0.03 , i.e. comparable to our estimates of $F m_{\text {extr }}$ values listed in Table 4. Thus, it appears that Alconox residue was largely responsible for the extraneous carbon mass in our isolates.

\section{CONCLUSIONS}

We have utilized small-scale CSRA by AMS to determine the biogenic fraction of a food contaminant in a fatty food matrix at concentrations well below $1 \mathrm{mg} / \mathrm{kg}$. The purity of the isolates prepared for AMS analysis was assessed, as well as the influence of extraneous contaminant carbon from all steps of the sample preparation and analysis. Our results indicate that the majority of DEHP extracted from the 2 Stilton cheese wheels is of an industrial, petrogenic nature. However, consistently higher-than-fossil values of corrected $F C$ indicate that a significant portion of DEHP present in the cheese is of modern, likely biogenic, origin. This is in accordance with and strongly supportive of previous studies suggesting that bis(2-ethylhexyl) phthalate is naturally produced by certain algae, mold, and, likely in this case, strains of penicillium.

\section{ACKNOWLEDGMENTS}

This work was performed with funding from the US Food and Drug Administration's Office of Food Additive Safety (Division of Food Contact Notification) under grant number U01FD001418; and in part under the auspices of the US Department of Energy by Lawrence Livermore National Laboratory under Contract DE-AC52-07NA27344. We thank Michele Schantz of the NIST Analytical Chemistry Division for providing guidance and training; and Professor Alan Kaufman, Dept. of Geology at the University of Maryland College Park, for performing ${ }^{13} \mathrm{C} /{ }^{12} \mathrm{C}$ isotope ratio measurements in his Stable Isotope Laboratory. Certain commercial equipment, instruments, or materials are identified in this paper to specify adequately the experimental procedure. Such identification does not imply recommendation or endorsement by the National Institute of Standards and Technology or the US Food and Drug Administration, nor does it imply that the materials or equipment identified are necessarily the best available for the purpose. 


\section{MA Nelson et al.}

\section{REFERENCES}

Amade P, Mallea M, Bouaicha N. 1994. Isolation, structural identification and biological activity of two metabolites produced by Penicillium Olsonii Bainier and Sartory. Journal of Antibiotics 47(2):201-7.

Brown TA, Southon JR. 1997. Corrections for contamination background in AMS ${ }^{14} \mathrm{C}$ measurements. $\mathrm{Nu}$ clear Instruments and Methods in Physics Research B 123(1-4):208-13.

Castle L, Gilbert J, Eklund T. 1990. Migration of plasticizer from poly(vinyl chloride) milk tubing. Food $\mathrm{Ad}$ ditives and Contaminants 7(5):591-6.

Chen CY. 2004. Biosynthesis of di-92-ethylhexyl) phthalate (DEHP) and di-n-butyl phthalate (DBP) from red alga - Bangia atropurpurea. Water Research 38(4): 1014-8.

David R. 2000. Human exposure estimates for phthalates: correspondence. Environmental Health Perspectives 108(10):A440-A442.

Enneking PA. 2006. Phthalates not in plastic food packaging. Environmental Health Perspectives 114(2): A89-A90.

European Council for Plasticisers and Intermediates (ECPI). 2012. DEHP Information Centre: About DEHP [Internet]. Accessed 13 September 2012. URL: http://www.dehp-facts.com/DEHP.

Grubbs FE. 1969. Procedures for detecting outlying observations in samples. Technometrics 11(1):1-21.

Jarosova A. 2006. Phthalic acid esters (PAEs) in the food chain. Czech Journal of Food Sciences 24(5):223-31.

Namikoshi M, Fujiwarea T, Nishikawa T, Ukai K. 2006. Natural abundance ${ }^{14} \mathrm{C}$ content of dibutyl phthalate (DBP) from three marine algae. Marine Drugs 4(4): 290-7.

Pearson A, McNichol AP, Benitez-Nelson BC, Hayes JM, Eglinton TI. 2001. Origins of lipid biomarkers in Santa Monica Basin surface sediment: a case study using compound specific ${ }^{14} \mathrm{C}$ analysis. Geochimica et Cosmochimica Acta 65(18):3123-37.

Reddy CM, Pearson A, Xu L, McNichol AP, Bennter Jr BA, Wiste SA, Klouda GA, Currie LA, Eglinton TI.
2002. Radiocarbon as a tool to apportion the sources of polycyclic aromatic hydrocarbons and black carbon in environmental samples. Environmental Science \& Technology 36(8):1774-82.

Reimer PJ, Brown TA, Reimer RW. 2004. Discussion: reporting and calibration of post-bomb ${ }^{14} \mathrm{C}$ data. Radiocarbon 46(3):1299-304.

Santos GM, Southon JR, Druffel-Rodriguez KC, Griffin S, Mazon M. 2004. Magnesium perchlorate as an alternative water trap in AMS graphite sample preparation: a report on sample preparation at KCCAMS at the University of California, Irvine. Radiocarbon 46(1):165-73.

Schettler T. 2006. Human exposure to phthalates via consumer products. International Journal of Andrology 29(1):134-5.

Stuiver M, Polach HA. 1977. Discussion: reporting of ${ }^{14} \mathrm{C}$ data. Radiocarbon 19(3):355-63.

US Environmental Protection Agency (US EPA). 1997. Integrated Risk Information System Database: Di(2ethylhexyl)phthalate (DEHP) [Internet]. CASRN 11781-7. Accessed 14 September 2012. URL: http:// www.epa.gov/IRIS/subst/0014.htm.

Vogel JS, Southon JR, Nelson DE. 1987. Catalyst and binder effects in the use of filamentous graphite for AMS. Nuclear Instruments and Methods in Physics Research B 29(1-2):50-6.

Wenzl T. 2009. Methods for the Determination of Phthalates in Food. JRC Scientific and Technical Reports, Institute for Reference Materials and Measurements, Joint Research Centre, European Commission, EUR23682 EN, Luxembourg.

Wormuth M, Scheringer M, Vollenweider M, Hungerbuhler K. 2006. What are the sources of exposure to eight frequently used phthalic acid esters in Europeans? Risk Analysis 26(3):803-24.

Zhu J, Phillips SP, Feng YL, Yang X. 2006. Phthalate esters in human milk: concentration variations over a 6 month postpartum time. Environmental Science \& Technology 40(17):5276-81. 